\title{
The General Traveling Wave Solutions of the Fisher Equation with Degree Three
}

\author{
Wenjun Yuan, ${ }^{1,2}$ Qiuhui Chen, ${ }^{3}$ Jianming $Q i,{ }^{4}$ and Yezhou $\mathrm{Li}^{5}$ \\ ${ }^{1}$ School of Mathematics and Information Science, Guangzhou University, Guangzhou 510006, China \\ ${ }^{2}$ Key Laboratory of Mathematics and Interdisciplinary Sciences of Guangdong Higher Education Institutes, \\ Guangzhou University, Guangzhou 510006, China \\ ${ }^{3}$ Cisco School of Informatics, Guangdong University of Foreign Studies, Guangzhou 510420, China \\ ${ }^{4}$ Department of Mathematics and Physics, Shanghai Dianji University, Shanghai 201306, China \\ ${ }^{5}$ School of Science, Beijing University of Posts and Telecommunications, Beijing 100876, China \\ Correspondence should be addressed to Wenjun Yuan; wjyuan1957@126.com and Jianming Qi; qijianmingdaxia@163.com
}

Received 13 May 2013; Accepted 19 August 2013

Academic Editor: Ricardo Weder

Copyright ( 2013 Wenjun Yuan et al. This is an open access article distributed under the Creative Commons Attribution License, which permits unrestricted use, distribution, and reproduction in any medium, provided the original work is properly cited.

We employ the complex method to research the integrality of the Fisher equations with degree three. We obtain the sufficient and necessary condition of the integrable of the Fisher equations with degree three and the general meromorphic solutions of the integrable Fisher equations with degree three, which improves the corresponding results obtained by Feng and Li (2006), Guo and Chen (1991), and Ağırseven and Öziş (2010). Moreover, all $w_{g, 1}(z)$ are new general meromorphic solutions of the Fisher equations with degree three for $c= \pm 3 / \sqrt{2}$. Our results show that the complex method provides a powerful mathematical tool for solving a large number of nonlinear partial differential equations in mathematical physics.

\section{Introduction and Main Result}

Consider the Fisher equation

$$
u_{t}=v u_{x x}+s u(1-u)
$$

which is a nonlinear diffusion equation as a model for the propagation of a mutant gene with an advantageous selection intensity $s$. It was suggested by Fisher as a deterministic version of a stochastic model for the spatial spread of a favored gene in a population in 1936.

Set $t^{\prime}=s t$ and $x^{\prime}=(s / v)^{1 / 2} x$ and drop the primes; (1) becomes

$$
u_{t}=u_{x x}+u(1-u) .
$$

Substituting the traveling wave transform $u(x, t)=w(z)$, $x-c t=z$ into (2), it gives a nonlinear ordinary differential equation

$$
w^{\prime \prime}+c w^{\prime}+w(1-w)=0,
$$

where $c$ is a constant.
Finding solutions of nonlinear models is a difficult and challenging task.

In 2005 and 2009, Feng et al. $[1,2]$ proposed an analytic method to construct explicitly exact and approximate solutions for nonlinear evolution equations. By using this method, some new traveling wave solutions of the KuramotoSivashinsky equation and the Benny equation were obtained explicitly. These solutions included solitary wave solutions, singular traveling wave solutions, and periodical wave solutions. These results indicated that in some cases their analytic approach is an effective method to obtain traveling solitary wave solutions of various nonlinear evolution equations. It can also be applied to some related nonlinear dynamical systems.

In 2010 and 2011, Demina et al. [3-5] studied the meromorphic solutions of autonomous nonlinear ordinary differential equations. An algorithm for constructing meromorphic solutions in explicit form was presented. General expressions for meromorphic solutions (including rational, periodic, elliptic) were found for a wide class of autonomous nonlinear ordinary differential equations. 
Recently, the complex method was introduced by Yuan et al. [6-8].

Very recently, Yuan et al. [9] employed the complex method to obtain the general solutions of (3). In order to state our results, we need some concepts and notations.

A meromorphic function $w(z)$ means that $w(z)$ is holomorphic in the complex plane $\mathbb{C}$ except for poles. Denote by $\wp\left(z ; g_{2}, g_{3}\right)$ the Weierstrass elliptic function with invariants $g_{2}$ and $g_{3}$. We say that a meromorphic function $f$ belongs to the class $W$ if $f$ is an elliptic function, or a rational function of $e^{\alpha z}, \alpha \in \mathbb{C}$, or a rational function of $z$.

Theorem 1. Equation (3) is integrable if and only if $c=0$, $\pm 5 / \sqrt{6}, \pm 5 i / \sqrt{6}$. Furthermore, the general solutions of (3) are of the following forms.

(I) (See [6]), when $c=0$, the elliptic general solutions of (3)

$$
w_{d}(z)=6\left\{-\wp(z)+\frac{1}{4}\left[\frac{\wp^{\prime}(z)+F}{\wp(z)-E}\right]^{2}\right\}-6 E+\frac{1}{2},
$$

where, $g_{2}=1 / 12, F^{2}=4 E^{3}-g_{2} E-g_{3}, g_{3}$ and $E$ are arbitrary. In particular, it degenerates the simply periodic solutions

$$
w_{s}(z)=6 \alpha^{2} \operatorname{coth}^{2} \frac{\alpha}{2}\left(z-z_{0}\right)+\frac{1}{2} \alpha^{2}+\frac{1}{2},
$$

where $\alpha^{4}=1, z_{0} \in \mathbb{C}$.

(II) (See [10]), when $c= \pm 5 / \sqrt{6}$, the general solutions of (3)

$$
w_{g}(z)=\exp \left\{\mp \frac{2 z}{\sqrt{6}}\right\} \wp\left(\exp \left\{\mp \frac{z}{\sqrt{6}}\right\}-s_{0} ; 0, g_{3}\right)
$$

where both $s_{0}$ and $g_{3}$ are arbitrary constants. In particular, it degenerates the one parameter family of solutions

$$
w_{f}(z)=\frac{1}{\left\{1-\exp \left\{ \pm\left(z-z_{0}\right) / \sqrt{6}\right\}\right\}^{2}},
$$

where $z_{0} \in \mathbb{C}$.

(III) (See [9]), when $c= \pm 5 i / \sqrt{6}$, the general solutions of (3) $w_{g, i}(z)=\exp \left\{\mp \frac{2 i z}{\sqrt{6}}\right\} \wp\left(i \exp \left\{\mp \frac{i z}{\sqrt{6}}\right\}-s_{0} ; 0, g_{3}\right)+1$,

where both $s_{0}$ and $g_{3}$ are arbitrary constants. In particular, it degenerates the one parameter family of solutions

$w_{f, i}(z)=-\frac{1}{\left\{1-\exp \left\{ \pm i\left(z-z_{0}\right) / \sqrt{6}\right\}\right\}^{2}}+1$,

where $z_{0} \in \mathbb{C}$.
Remark 2. The Fisher equation is a classic and the simplest case of the nonlinear reaction-diffusion equation, but there are many applications about it and many authors have been researching it (cf. [11]). The first explicit form of a traveling wave solution for the Fisher equation was obtained by Ablowitz and Zeppetella [10] using the Painleve analysis. three

In this paper, we consider the Fisher equation with degree

$$
w^{\prime \prime}+c w^{\prime}+w\left(1-w^{2}\right)=0
$$

where $c$ is a constant.

Our main result is the following theorem.

Theorem 3. Equation (10) is integrable if and only if $c=0$, $\pm 3 / \sqrt{2}$. Furthermore, the general solutions of (10) are of the following form.

(I) (See [12]), when $c=0$, the elliptic general solutions of (10)

$$
w_{d, 1}(z)= \pm \frac{\sqrt{2}}{2} \frac{\wp^{\prime}\left(z-z_{0}: g_{2}, 0\right)}{\wp\left(z-z_{0}: g_{2}, 0\right)}
$$

where $z_{0}$ and $g_{2}$ are arbitrary. In particular, it degenerates the simply periodic solutions

$$
w_{s, 1}(z)= \pm \sqrt{2} \tanh \left(z-z_{0}\right)
$$

where $z_{0} \in \mathbb{C}$.

(II) When $c= \pm 3 / \sqrt{2}$, the general solutions of (10)

$$
w_{g, 1}(z)= \pm \frac{1}{2} \exp \left\{\mp \frac{z}{\sqrt{2}}\right\} \frac{\wp^{\prime}\left(\exp \{\mp z / \sqrt{2}\}-s_{0} ; g_{2}, 0\right)}{\wp\left(\exp \{\mp z / \sqrt{2}\}-s_{0} ; g_{2}, 0\right)}
$$

where $\wp\left(s: g_{2}, 0\right)$ is the Weierstrass elliptic function, both $s_{0}$ and $g_{2}$ are arbitrary constants. In particular, $w_{g, 1}(z)$ degenerates the one parameter family of solutions

$$
w_{f, 1}(z)= \pm \frac{1}{1-\exp \left\{ \pm\left(z-z_{0}\right) / \sqrt{2}\right\}},
$$

where $z_{0} \in \mathbb{C}$.

Remark 4. The Fisher equation is a classic and the simplest case of the nonlinear reaction-diffusion equation, but there are many applications about it and many authors have been researching it (cf. [13]). Many authors obtained only $w_{f, 1}(z)$ by using other methods (cf. [13]). Moreover, all $w_{g, 1}(z)$ are new general meromorphic solutions of the Fisher equations with degree three for $c= \pm 3 / \sqrt{2}$.

Remark 5. The growth order of each $w_{g}(z), w_{g, i}(z), w_{g, 1}(z)$ is infinite. 
This paper is organized as follows. In Section 2, the preliminary lemmas and the complex method are given. The proof of Theorem 3 is given and the general meromorphic solutions of (10) are derived by complex method in Section 3. Some conclusions and discussions are given in the final section.

\section{Preliminary Lemmas and the Complex Method}

In order to give our complex method and the proof of Theorem 1 , we need some notations and results.

Set $m \in \mathbb{N}:=\{1,2,3, \ldots\}, r_{j} \in \mathbb{N}_{0}=\mathbb{N} \cup\{0\}, r=$ $\left(r_{0}, r_{1}, \ldots, r_{m}\right), j=0,1, \ldots, m$. We define a differential monomial denoted by

$$
M_{r}[w](z):=[w(z)]^{r_{0}}\left[w^{\prime}(z)\right]^{r_{1}}\left[w^{\prime \prime}(z)\right]^{r_{2}} \cdots\left[w^{(m)}(z)\right]^{r_{m}} .
$$

And $p(r):=r_{0}+r_{1}+\cdots+r_{m}$ is called the degree of $M_{r}[w]$. A differential polynomial is defined by

$$
P\left(w, w^{\prime}, \ldots, w^{(m)}\right):=\sum_{r \in I} a_{r} M_{r}[w],
$$

where $a_{r}$ are constants, and $I$ is a finite index set. The total degree is defined by $\operatorname{deg} P\left(w, w^{\prime}, \ldots, w^{(m)}\right):=\max _{r \in I}\{p(r)\}$.

We will consider the following complex ordinary differential equations:

$$
P\left(w, w^{\prime}, \ldots, w^{(m)}\right)=b w^{n}+c,
$$

where $b \neq 0, c$ are constants, $n \in \mathbb{N}$.

Let $p, q \in \mathbb{N}$. Suppose that (17) has a meromorphic solution $w$ with at least one pole; we say that (17) satisfies weak $\langle p, q\rangle$ condition if by substituting Laurent series

$$
w(z)=\sum_{k=-q}^{\infty} c_{k} z^{k}, \quad q>0, c_{-q} \neq 0
$$

into (17), we can determin $p$ distinct Laurent singular parts below

$$
\sum_{k=-q}^{-1} c_{k} z^{k}
$$

In order to give the representations of elliptic solutions, we need some notations and results concerning elliptic function [14].

Let $\omega_{1}, \omega_{2}$ be two given complex numbers such that Im $\omega_{1} / \omega_{2}>0, L=L\left[2 \omega_{1}, 2 \omega_{2}\right]$ be discrete subset $L\left[2 \omega_{1}, 2 \omega_{2}\right]=\left\{\omega \mid \omega=2 n \omega_{1}+2 m \omega_{2}, n, m \in \mathbb{Z}\right\}$, which is isomorphic to $\mathbb{Z} \times \mathbb{Z}$. The discriminant $\Delta=\Delta\left(c_{1}, c_{2}\right):=$ $c_{1}^{3}-27 c_{2}^{2}$ and

$$
s_{n}=s_{n}(L):=\sum_{\omega \in L \backslash\{0\}} \frac{1}{\omega^{n}} .
$$

Weierstrass elliptic function $\wp(z):=\wp\left(z, g_{2}, g_{3}\right)$ is a meromorphic function with double periods $2 \omega_{1}, 2 \omega_{2}$ and satisfying the equation

$$
\left(\wp^{\prime}(z)\right)^{2}=4 \wp(z)^{3}-g_{2} \wp(z)-g_{3},
$$

where $g_{2}=60 s_{4}, g_{3}=140 s_{6}$ and $\Delta\left(g_{2}, g_{3}\right) \neq 0$.

Lemma 6 (see $[14,15])$. Weierstrass elliptic functions $\wp(z):=$ $\wp\left(z, g_{2}, g_{3}\right)$ have two successive degeneracies and addition formula.

(I) Degeneracy to simply periodic functions (i.e., rational functions of one exponential $e^{k z}$ ) according to

$$
\wp\left(z, 3 d^{2},-d^{3}\right)=2 d-\frac{3 d}{2} \operatorname{coth}^{2} \sqrt{\frac{3 d}{2}} z,
$$

if one root $e_{j}$ is double $\left(\Delta\left(g_{2}, g_{3}\right)=0\right)$.

(II) Degeneracy to rational functions of $z$ according to

$$
\wp(z, 0,0)=\frac{1}{z^{2}}
$$

if one root $e_{j}$ is triple $\left(g_{2}=g_{3}=0\right)$.

(III) Addition formula

$$
\wp\left(z-z_{0}\right)=-\wp(z)-\wp\left(z_{0}\right)+\frac{1}{4}\left[\frac{\wp^{\prime}(z)+\wp^{\prime}\left(z_{0}\right)}{\wp(z)-\wp\left(z_{0}\right)}\right]^{2} .
$$

In the proof of our main result, the following lemmas are very useful, which can be deduced by Theorem 1 in [12].

Lemma 7 (see [12]). The differential equation

$$
w^{\prime \prime}+w-w^{3}=0
$$

has the elliptic solutions and simply periodic solution with pole at $z=0$

$$
\begin{gathered}
w(z)= \pm \frac{\sqrt{2}}{2} \frac{\wp^{\prime}\left(z: g_{2}, 0\right)}{\wp\left(z: g_{2}, 0\right)}, \\
w(z)= \pm \sqrt{2} \tanh z,
\end{gathered}
$$

respectively, where $\wp\left(z: g_{2}, 0\right)$ is the Weierstrass elliptic function with $g_{3}=0$ and $g_{2}$ arbitrary.

Lemma 8 (see [12]). The differential equation

$$
w^{\prime \prime}=2 w^{3}
$$

has the elliptic solutions with pole at $z=0$

$$
w(z)= \pm \frac{1}{2} \frac{\wp^{\prime}\left(z: g_{2}, 0\right)}{\wp\left(z: g_{2}, 0\right)},
$$

where $\wp\left(z: g_{2}, 0\right)$ is the Weierstrass elliptic function with $g_{3}=$ 0 and $g_{2}$ arbitrary. 
By the aforementioned lemmas and results, we can give a new method below, say complex method, to find exact solutions of some PDEs.

Step 1. Substituting the transform $T: u(x, t) \rightarrow w(z)$, $(x, t) \rightarrow z$ into a given PDE gives a nonlinear ordinary differential equations (17).

Step 2. Substitute (18) into (17) to determine that weak $\langle p, q\rangle$ condition holds and pass the Painlevé test for (17).

Step 3. Find the meromorphic solutions $w(z)$ of (17) with pole at $z=0$, which have $m-1$ integral constants.

Step 4. By the addition formula of Lemma 6 we obtain the general meromorphic solutions $w\left(z-z_{0}\right)$.

Step 5. Substituting the inverse transform $T^{-1}$ into these meromorphic solutions $w\left(z-z_{0}\right)$, then we get all exact solutions $u(x, t)$ of the original given PDE.

\section{Proof of Theorem 3}

Proof. Substituting (18) into (10) we have $q=1, p=2, c_{-1}=$ $\pm \sqrt{2}, c_{0}=\mp c / 3 \sqrt{2}, c_{1}= \pm\left(6-c^{2}\right) / 18 \sqrt{2}, c_{2}=0$, and

$$
0 \times c_{3}+\left(\frac{c}{3}\right)^{2}-2\left(\frac{c}{3}\right)^{4}=0
$$

For the Laurent expansion (18) to be valid $c$ satisfies this equation and $c_{3}$ is an arbitrary constant. Therefore, $c=$ $0, \pm 3 / \sqrt{2}$. For other $c$ it would be necessary to add logarithmic terms to the expansion, thus giving a branch point rather than a pole.

For $c=0,(10)$ is completely integrable by standard techniques and the solutions are expressible in terms of elliptic functions (cf. [12]). That is, by Lemmas 6 and 7, the elliptic general solutions of (10)

$$
w_{d, 1}(z)= \pm \frac{\sqrt{2}}{2} \frac{\wp^{\prime}\left(z-z_{0}: g_{2}, 0\right)}{\wp\left(z-z_{0}: g_{2}, 0\right)}
$$

where $z_{0}$ and $g_{2}$ are arbitrary. In particular, it degenerates the simply periodic solutions

$$
w_{s, 1}(z)= \pm \sqrt{2} \tanh \left(z-z_{0}\right)
$$

where $z_{0} \in \mathbb{C}$.

For $c= \pm 3 / \sqrt{2}$, we transform (10) into the second Painlevé type equation. In this way we find the general solutions.

Setting $w(z)=f(z) u(s), s=g(z)$ and substituting in Fisher's equation (10), we obtain that the equation for $u(s)$ is

$$
\begin{aligned}
\left(g^{\prime}\right)^{2} u^{\prime \prime}= & -u^{\prime} g^{\prime}\left\{c+\frac{2 f^{\prime}}{f}+\frac{g^{\prime \prime}}{g^{\prime}}\right\} \\
& -\left\{1+c \frac{f^{\prime}}{f}+\frac{f^{\prime \prime}}{f}\right\} u+f^{2} u^{3} .
\end{aligned}
$$

If we take $f$ and $g$ such that

$$
\begin{aligned}
& c+\frac{2 f^{\prime}}{f}+\frac{g^{\prime \prime}}{g^{\prime}}=0, \\
& 1+c \frac{f^{\prime}}{f}+\frac{f^{\prime \prime}}{f}=0,
\end{aligned}
$$

then (32) for $u$ is integrable. By (33), one takes $f(z)=\exp \alpha z$ and

$$
g(z)=\beta \exp \{-(c+2 \alpha) z\},
$$

where $\alpha=\mp 1 / \sqrt{2}, \beta^{2}=1$.

Thus (32) reduces to

$$
u^{\prime \prime}=2 u^{3}
$$

Both Lemmas 6 and 8 give that the general solutions of (35) are of the form

$$
u(s)= \pm \frac{1}{2} \frac{\wp^{\prime}\left(s-s_{0} ; g_{2}, 0\right)}{\wp\left(s-s_{0} ; g_{2}, 0\right)},
$$

where $\wp(s)$ is the Weierstrass elliptic function $s_{0}$ and $g_{2}$ are two arbitrary constants.

Therefore, when $c= \pm 3 / \sqrt{2}$, by Lemma 6 , the general solutions of (10)

$$
w_{g, 1}(z)= \pm \frac{1}{2} \exp \left\{\mp \frac{z}{\sqrt{2}}\right\} \frac{\wp^{\prime}\left(\exp \{\mp z / \sqrt{2}\}-s_{0} ; g_{2}, 0\right)}{\wp\left(\exp \{\mp z / \sqrt{2}\}-s_{0} ; g_{2}, 0\right)}
$$

where both $s_{0}$ and $g_{2}$ are arbitrary constants. In particular, by Lemma 6 and $g_{2}=0, w_{g, 1}(z)$ degenerates the one parameter family of solutions

$$
w_{f, 1}(z)= \pm \frac{1}{1-\exp \left\{ \pm\left(z-z_{0}\right) / \sqrt{2}\right\}}
$$

where $z_{0} \in \mathbb{C}$.

\section{Conclusions}

Complex method is a very important tool in finding the exact solutions of nonlinear evolution equations, and the general Fisher equations are simplest case of the nonlinear reactiondiffusion equations. In this paper, we employ the complex method to research the integrality of the Fisher equations with degree three. We obtain the sufficient and necessary condition of integrable of the Fisher equations with degree three and the general meromorphic solutions of the integrable Fisher equations with degree three, which improves the corresponding results obtained by Feng and Li [11], Guo and Chen [16], and Ağırseven and Öziş [13]. Moreover, all $w_{g, 1}(z)$ are new general meromorphic solutions of the Fisher equations with degree three for $c= \pm 3 / \sqrt{2}$. Our results show that the complex method provides a powerful mathematical tool for solving a large number of nonlinear partial differential equations in mathematical physics. 


\section{Acknowledgments}

This work was supported by the Visiting Scholar Program of Chern Institute of Mathematics at Nankai University when the authors worked as Visiting Scholars. This work was completed with the support of the NSF of China (11271090, 11101048, and 11171184) and NSF of Guangdong Province (S2012010010121) and Shanghai University Young Teacher Training Program (ZZSDJ12020), projects 10XKJ01, 12C401, and 12C104, from the Leading Academic Discipline Project of Shanghai Dianji University.

\section{References}

[1] Z. Feng, S. Zheng, and D. Y. Gao, "Traveling wave solutions to a reaction-diffusion equation," Zeitschrift für Angewandte Mathematik und Physik, vol. 60, no. 4, pp. 756-773, 2009.

[2] Z. Feng and G. Chen, "Traveling wave behavior for a nonlinear reaction-diffusion equation," Dynamics of Continuous, Discrete \& Impulsive Systems. Series A, vol. 12, no. 5, pp. 643-664, 2005.

[3] M. V. Demina and N. A. Kudryashov, "From Laurent series to exact meromorphic solutions: the Kawahara equation," Physics Letters A, vol. 374, no. 39, pp. 4023-4029, 2010.

[4] M. V. Demina and N. A. Kudryashov, "Explicit expressions for meromorphic solutions of autonomous nonlinear ordinary differential equations," Communications in Nonlinear Science and Numerical Simulation, vol. 16, no. 3, pp. 1127-1134, 2011.

[5] N. A. Kudryashov, D. I. Sinelshchikov, and M. V. Demina, "Exact solutions of the generalized Bretherton equation," Physics Letters A, vol. 375, no. 7, pp. 1074-1079, 2011.

[6] W. J. Yuan, Y. Z. Li, and J. M. Lin, "Meromorphic solutions of an auxiliary ordinary differential equation using complex method," Mathematical Methods in the Applied Sciences, vol. 36, no. 13, pp. 1776-1782, 2013.

[7] W. J. Yuan, Y. Huang, and Y. D. Shang, "All traveling wave exact solutions of two nonlinear physical models," Applied Mathematics and Computation, vol. 219, no. 11, pp. 6212-6223, 2013.

[8] W. J. Yuan, Y. D. Shang, Y. Huang, and H. Wang, “The representation of meromorphic solutions to certain ordinary differential equations and its applications," Scientia Sinica Mathematica, vol. 43, no. 6, pp. 563-575, 2013.

[9] W. J. Yuan, Q. H. Chen, W. L. Xiong, and J. M. Qi, “The general meromorphic solutions of the Fisher equation," Physics Letters A. In press.

[10] M. J. Ablowitz and A. Zeppetella, "Explicit solutions of Fisher's equation for a special wave speed," Bulletin of Mathematical Biology, vol. 41, no. 6, pp. 835-840, 1979.

[11] Z. Feng and Y. Li, "Complex traveling wave solutions to the Fisher equation,” Physica A, vol. 366, pp. 115-123, 2006.

[12] W. J. Yuan, W. L. Xiong, J. M. Lin, and Y. H. Wu, "All meromorphic solutions of an auxiliary ordinary differential equation and its applications," Acta Mathematica Scientia. In press.

[13] D. Ağırseven and T. Öziş, "An analytical study for Fisher type equations by using homotopy perturbation method," Computers \& Mathematics with Applications, vol. 60, no. 3, pp. 602-609, 2010.

[14] S. Lang, Elliptic Functions, vol. 112 of Graduate Texts in Mathematics, Springer, New York, NY, USA, 2nd edition, 1987.

[15] R. Conte and M. Musette, "Elliptic general analytic solutions," Studies in Applied Mathematics, vol. 123, no. 1, pp. 63-81, 2009.

[16] B. Y. Guo and Z. X. Chen, "Analytic solutions of the Fisher equation," Journal of Physics A, vol. 24, no. 3, pp. 645-650, 1991. 


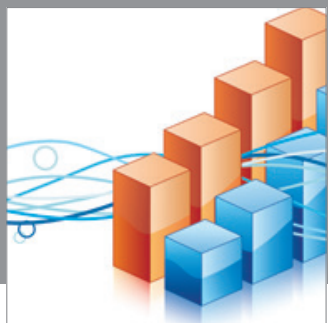

Advances in

Operations Research

mansans

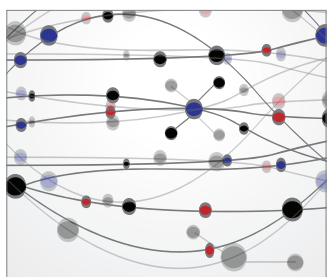

The Scientific World Journal
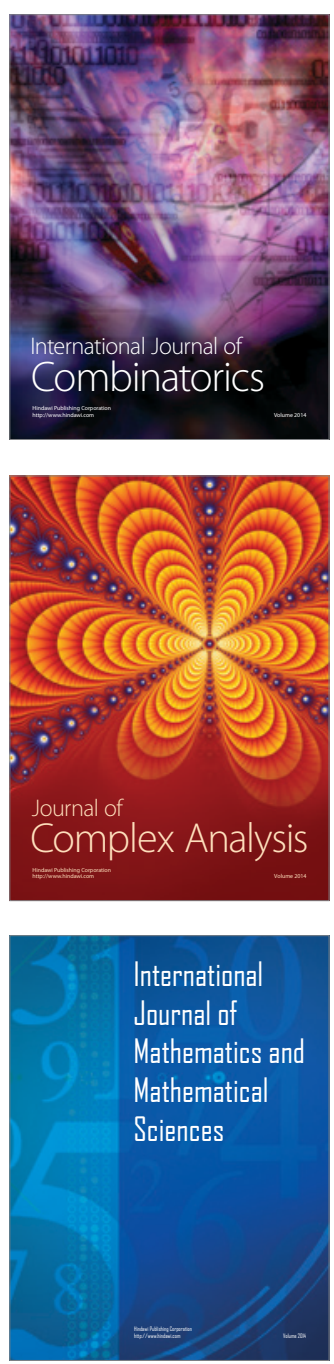
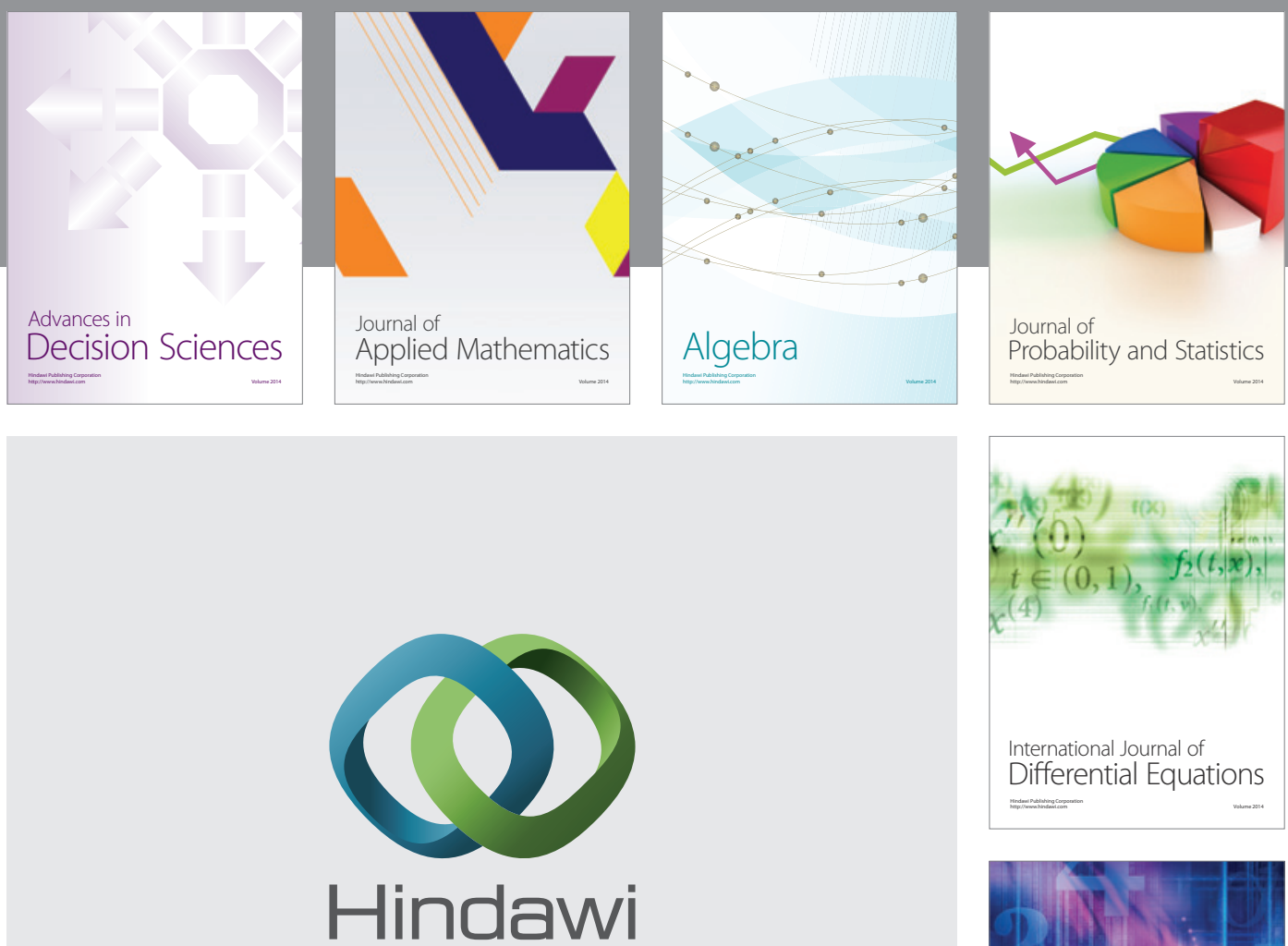

Submit your manuscripts at http://www.hindawi.com
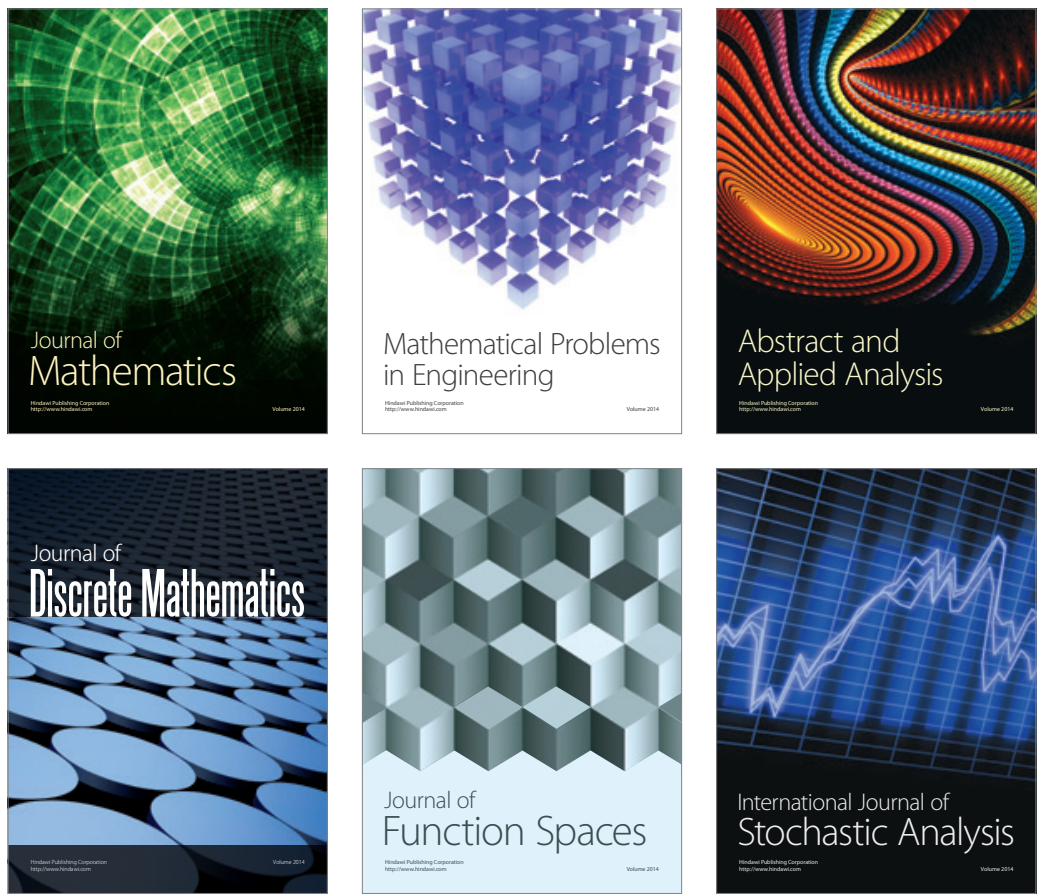

Journal of

Function Spaces

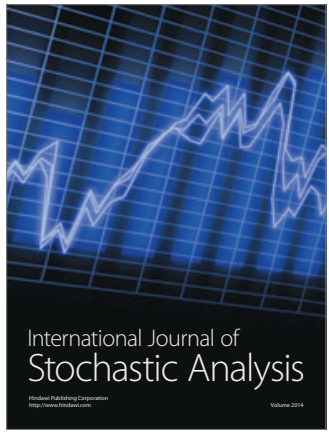

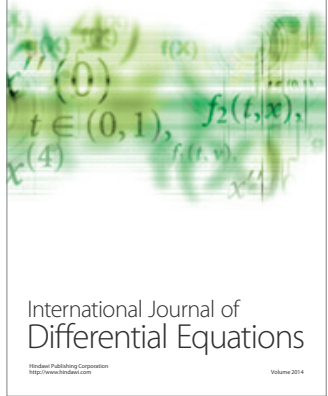
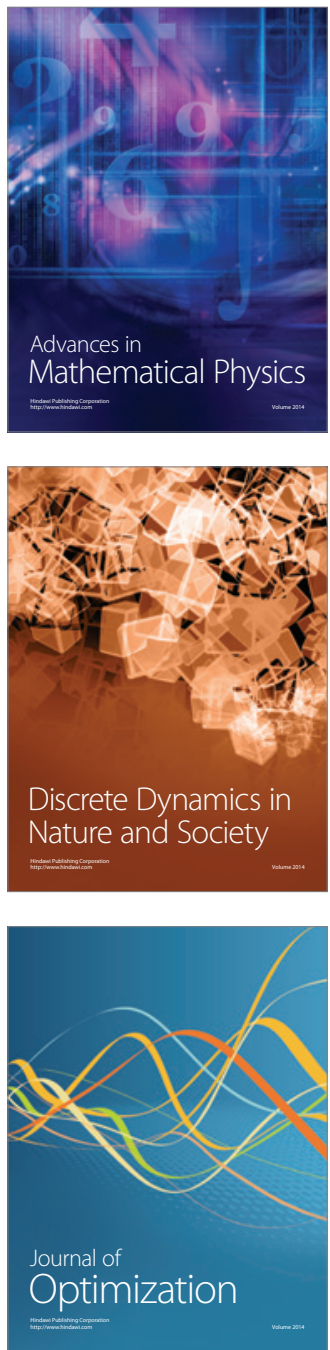